\title{
Continued fractions built from convex sets and convex functions
}

\author{
Ilya Molchanov* \\ Institute of Mathematical Statistics and Actuarial Science \\ University of Bern, Sidlerstrasse 5, 3012 Bern, Switzerland \\ ilya.molchanov@stat.unibe.ch
}

\begin{abstract}
In a partially ordered semigroup with the duality (or polarity) transform, it is possible to define a generalisation of continued fractions. General sufficient conditions for convergence of continued fractions are provided. Two particular applications concern the cases of convex sets with the Minkowski addition and the polarity transform and the family of non-negative convex functions with the Legendre-Fenchel and ArtsteinAvidan-Milman transforms.

AMS Classification: 46B10, 06F05, 11J70, 20M14, 26B25, 44A15, 52A22, 52A41

Keywords: continued fraction, semigroup, duality, polarity, convex body, convex function, Legendre-Fenchel transform, partial order
\end{abstract}

\section{Introduction}

The studies of order reversing involutions (also called dualities or polarities) on partially ordered spaces have recently gained a considerable attention. If $\mathbb{K}$ is a partially ordered space, then the map from $x \in \mathbb{K}$ to $x^{*} \in \mathbb{K}$ is said to be an order reversing involution if $x^{* *}=x$ for all $x$ and $x \leq y$ implies that $y^{*} \leq x^{*}$.

The two main examples are the family of convex sets containing the origin and ordered by inclusion and the family of convex functions on $\mathbb{R}^{d}$ ordered pointwisely. It is shown in [1, 5] that the only (up to a rigid motion) order reversing involution on the family of compact convex sets containing the origin is the classical polar transform, see [11] and Section 4. For the family of non-negative convex functions on $\mathbb{R}^{d}$ that vanish at the origin, only two involutions (up to rigid motions) exist: one is the classical Legendre-Fenchel transform [9] and the other is the A-transform, see [2, 3] and Section 5.

\footnotetext{
${ }^{*}$ Supported by the Swiss National Science Foundation grant 200021-137527 and the Santander Bank through the Chair of Excellence programme at the University Carlos III of Madrid.
} 
It is also possible to endow the space $\mathbb{K}$ with an addition operation that turns it into an abelian semigroup. Such an addition may be chosen to be the lattice operation corresponding to the order or defined otherwise. For example, on the family of closed convex sets partially ordered by inclusion it is possible to consider the convex hull of the union as the semigroup operation or add sets using the Minkowski (elementwise) addition. In the case of convex functions, a natural semigroup operation is the arithmetic addition, while it is also possible to consider the epigraphical or level sums. In this paper, it is assumed that $\mathbb{K}$ is an abelian semigroup with the additive operation that is consistent with the order.

The order reversing nature of the involution makes possible to define a continued fraction on a semigroup. In comparison with classical numerical continued fractions, the additive operation is the semigroup addition, while the one over operation is replaced by the involution. The classical concept of continued fraction is recovered for the semigroup $[0, \infty]$ with the conventional addition and the involution given by the arithmetic inverse. The semigroup setting differs from the setting of continued fractions in Jordan algebras pursued in 4 and the studies of multidimensional continued fractions in [7] and [8].

This paper argues that convergence results for continued fractions built from convex sets and those from convex functions can be derived from general statements concerning continued fractions on semigroups. The non-existence of an inverse operation to the addition renders impossible the direct use of most of the tools from the classical theory of continued fractions, see [6]. Instead, the key emphasis is put on the partial order together with bounds on the Lipschitz constant for the involution transform. These bounds are needed for properly chosen subsets of the original semigroup, e.g. for convex sets that contain the unit ball. The latter in the abstract setting becomes an involution-invariant element, which is also the key ingredient to define a suitable metric. In this very general setting, several sufficient conditions for convergence of continued fractions with constant and variable terms are obtained in Section 3. A particular attention is devoted to periodic continued fractions, whose limits may be regarded as a generalisation of quadratic irrational numbers in the semigroup setting.

The general results are applied for set-valued continued fractions in Section 4. For example, a continued fraction with a constant term being a set $K$ converges if $K$ is sandwiched between two Euclidean balls with diameters $r$ and $R$ such that either $r>1$, or $r=1$ and $R$ is finite, or $r<1$ and $R<r /(1-r)$, see Theorem 4.1. In the set-valued case, we obtain a necessary and sufficient condition for the convergence that amounts to the fact that an oddnumbered approximant of the continued fraction is a subset of the ball with radius strictly less than one. The key argument is the Lipschitz property of the polarity transform meaning that the Hausdorff distance between the polars of two convex sets containing a centred ball of radius $r$ is bounded by $r^{-2}$ times the Hausdorff distance between the original sets.

Section 5 presents several convergence results for continued fractions of convex functions both for the Legendre-Fenchel and A-transforms. It is rather easy to modify these results to apply to the semigroup of log-concave functions with the multiplication. Another possible application left outside of the framework of this paper is for the semigroup of probability measures with the convolution operation as the addition and the involution inherited by an application of the involution operation on the original space. 


\section{Continued fractions on semigroups}

Let $\mathbb{K}$ be a partially ordered abelian semigroup with the neutral element e. Assume that $\mathbf{e} \leq x$ for all $x \in \mathbb{K}$ and that the order is compatible with the addition, that is $x \leq y$ for $x, y \in \mathbb{K}$ implies $x+z \leq y+z$ for all $z \in \mathbb{K}$. Assume that, the order is weaker than the semigroup order, i.e. $y=x+z$ for some $z \in \mathbb{K}$ yields that $x \leq y$. In this case, the semigroup is reduced, meaning that the only invertible element is $\mathbf{e}$.

Assume that $\mathbb{K}$ is equipped with an order reversing involution $x \mapsto x^{*}$ (also called duality or polarity transform), so that $x^{* *}=x$ and $x \leq y$ implies that $x^{*} \geq y^{*}$. The involution is not assumed to commute with the addition, i.e. $(x+y)^{*}$ is not necessarily $x^{*}+y^{*}$. Since $\mathbf{e} \leq x$ for each $x \in \mathbb{K}$, we have $x^{*} \leq \mathbf{e}^{*}$ meaning that $\mathbf{e}^{*}$ dominates all elements from $\mathbb{K}$ and $\mathbf{e}^{*}+x=\mathbf{e}^{*}$ for all $x \in \mathbb{K}$.

Consider a sequence $\left\{x_{n}, n \geq 1\right\}$ of elements from $\mathbb{K}$ and define the sequence $\left[x_{1}, \ldots, x_{n}\right]$, $n \geq 1$, recursively by letting

$$
\begin{aligned}
{\left[x_{1}\right] } & =x_{1}^{*} \\
{\left[x_{1}, \ldots, x_{n+1}\right] } & =\left(x_{1}+\left[x_{2}, \ldots, x_{n+1}\right]\right)^{*}, \quad n \geq 1 .
\end{aligned}
$$

The element $z_{n}=\left[x_{1}, \ldots, x_{n}\right]$ is said to be the $n$th approximant of the continued fraction generated by $\left\{x_{n}, n \geq 1\right\}$.

Remark 2.1. The dual operation to the addition is defined by setting

$$
x \oplus y=\left(x^{*}+y^{*}\right)^{*} .
$$

Then $\left[x_{1}, \ldots, x_{n}\right]=x_{1}^{*} \oplus\left[x_{2}, \ldots, x_{n}\right]^{*}$.

Example 2.2. If $\mathbb{K}=[0, \infty]$ with the conventional addition and involution $x^{*}=\frac{1}{x}$, then

$$
z_{n}=\left[x_{1}, \ldots, x_{n}\right]=\frac{1}{x_{1}+\frac{1}{x_{2}+\frac{1}{x_{3}+\cdots+\frac{1}{x_{n}}}}}
$$

is the $n$th approximant of the numerical continued fraction, see e.g. [6].

Remark 2.3. The neutral element and its dual influence the continued fraction as follows

$$
\begin{aligned}
{\left[x_{1}, \ldots, x_{m}, \mathbf{e}^{*}, x_{m+1}, \ldots, x_{n}\right] } & =\left[x_{1}, \ldots, x_{m}\right] \\
{\left[x_{1}, \ldots, x_{m}, \mathbf{e}, x_{m+1}, \ldots, x_{n}\right] } & =\left[x_{1}, \ldots, x_{m}+x_{m+1}, \ldots, x_{n}\right], \quad m \neq 0, n, \\
{\left[\mathbf{e}, x_{1}, \ldots, x_{n}\right] } & =\left[x_{1}, \ldots, x_{n}\right]^{*}=x_{1}+\left[x_{2}, \ldots, x_{n}\right], \\
{\left[x_{1}, \ldots, x_{n}, \mathbf{e}\right] } & =\left[x_{1}, \ldots, x_{n-1}\right] .
\end{aligned}
$$

The following result generalises the well-known property of continued fractions with positive terms.

Lemma 2.4. The $n$th approximant $z_{n}=\left[x_{1}, \ldots, x_{n}\right]$ is increasing in each of the even numbered terms and decreasing in each of the odd numbered terms. The sequence $\left\{z_{2 m}, m \geq 1\right\}$ is increasing and $\left\{z_{2 m-1}, m \geq 1\right\}$ is decreasing. 
Proof. A direct check shows that $\left[x_{1}, \ldots, x_{k}, \ldots, x_{n}\right]$ is increasing in $x_{k}$ if $k$ is even and decreasing if $k$ is odd. Then

$$
z_{2 m+2}=\left[x_{1}, \ldots, x_{2 m},\left(x_{2 m+1}+x_{2 m+2}^{*}\right)^{*}\right] \geq\left[x_{1}, \ldots, x_{2 m}, \mathbf{e}^{*}\right]=\left[x_{1}, \ldots, x_{2 m}\right]=z_{2 m} .
$$

A similar argument applies to the odd part of the continued fraction.

From now on, assume that $\mathbb{K}$ is equipped with the scaling transformation $x \mapsto$ ax by positive real numbers $a$. It is assumed that the scaling satisfies the distributivity laws and that $(a x)^{*}=a^{-1} x^{*}$ for all $a>0$ and $x \in \mathbb{K}$. In particular, the second distributivity law implies that $a x \leq b x$ for $a \leq b$.

Fix any $h \in \mathbb{K}$ such that $h^{*}=h$ (in this case $h$ is said to be self-polar) and define, for $x, y \in \mathbb{K}$,

$$
\rho_{h}(x, y)=\inf \{t \geq 0: x \leq y+t h, y \leq x+t h\} .
$$

Note that $\rho_{h}$ is a semimetric that might take infinite values and is scale-homogeneous, that is $\rho_{h}(a x, a y)=a \rho_{h}(x, y)$ for $a>0$. The (possibly infinite) norm of $x \in \mathbb{K}$ is defined as $\|x\|_{h}=\rho_{h}(x, \mathbf{e})$.

Let $\mathbb{K}_{h}$ be the family of $x \in \mathbb{K}$ such that $x \leq a h$ for some $a>0$. Note that $\mathbb{K}_{h}$ is a sub-semigroup of $\mathbb{K}$. Since $\rho_{h}(x, y) \leq a$ for $x \leq a h$ and $y \leq a h, \rho_{h}(x, y)$ takes finite values for $x, y \in \mathbb{K}_{h}$. If $x \in \mathbb{K}_{h}$, then $\rho_{h}\left(a_{n} x, \mathbf{e}\right) \rightarrow 0$ as $a_{n} \downarrow 0$. Denote

$$
\mathbb{K}_{h}^{*}=\left\{x \in \mathbb{K}: x^{*} \in \mathbb{K}_{h}\right\} .
$$

It is sensible to let $0 h=\mathbf{e}$ and $\infty h=\mathbf{e}^{*}$.

In the following we assume that

$$
\bigcap_{t>0}\{x \in \mathbb{K}: x \leq y+t h\}=\{x \in \mathbb{K}: x \leq y\} .
$$

Lemma 2.5. If (2) holds, then $\rho_{h}$ is a metric on $\mathbb{K}_{h}$ and the order is closed, that is the set $\{(x, y): x \leq y\}$ is closed in the product space $\left(\mathbb{K}_{h}, \rho_{h}\right) \times\left(\mathbb{K}_{h}, \rho_{h}\right)$.

Proof. Because of (2), $\rho_{h}(x, y)=0$ yields that $x \leq y$ and $y \leq x$, so that $x=y$. Other properties of the metric are evidently satisfied. If $\rho_{h}\left(x_{n}, x\right) \rightarrow 0$ and $\rho_{h}\left(y_{y}, y\right) \rightarrow 0$ and $x_{n} \leq y_{n}$ for all $n$, then for a sequence $t_{n} \rightarrow 0$ one has

$$
x \leq x_{n}+t_{n} h \leq y_{n}+t_{n} h \leq y+2 t_{n} h,
$$

so that $x \leq y$ by (2).

Remark 2.6 (Multiple self-polar elements). If $h_{1}$ and $h_{2}$ are two distinct self-polar elements, then $h_{1} \leq a h_{2}$ either holds for some $a>1$ or does not hold for any $a>0$. Indeed, by passing to the polars, the inequality becomes $h_{1} \geq a^{-1} h_{2}$, so that $a^{-1} h_{2} \leq h_{1} \leq a h_{2}$. If $h_{1} \leq a h_{2}$, then $a^{-1} \rho_{h_{1}}(x, y) \leq \rho_{h_{2}}(x, y) \leq a \rho_{h_{1}}(x, y)$ for all $x, y \in \mathbb{K}$. 
Definition 2.7. The continued fraction generated by a sequence $x_{n} \in \mathbb{K}, n \geq 1$, is said to converge if $z_{n}=\left[x_{1}, \ldots, x_{n}\right]$ converges in $\rho_{h}$ as $n \rightarrow \infty$ to an element of $\mathbb{K}$.

Example 2.8. If $x_{n}=b_{n} h$ for a self-polar $h \in \mathbb{K}$ and non-negative real numbers $b_{n}, n \geq 1$, then $z_{n}=\left[b_{1}, \ldots, b_{n}\right] h$, so that the convergence of $z_{n}$ can be derived from the convergence of the numerical continued fractions. For instance, the Seidel-Stern theorem asserts that $z_{n}$ converges if and only if $\sum b_{n}=\infty$. While it is tempting to conjecture that the continued fraction in $\mathbb{K}$ converges if $\sum x_{n}=\mathbf{e}^{*}$, Example 4.6 shows that this is wrong.

Lemma 2.9. Let $r_{n} h \leq x_{n} \leq R_{n} h, n \geq 1$, for $h \in \mathbb{K}$ such that $h^{*}=h$. Then

$$
\left[R_{1}, r_{2}, \ldots, a_{n}\right] h \leq\left[x_{1}, \ldots, x_{n}\right] \leq\left[r_{1}, R_{2}, \ldots, b_{n}\right] h,
$$

where $a_{n}=r_{n}$ and $b_{n}=R_{n}$ if $n$ is even and $a_{n}=R_{n}$ and $b_{n}=r_{n}$ if $n$ is odd.

Proof. It suffices to use the induction argument based on

$$
\begin{aligned}
{\left[R_{1} h, x_{2}, \ldots, x_{n}\right]=\left(R_{1} h+\left[x_{2}, \ldots, x_{n}\right]\right)^{*} } & \leq\left[x_{1}, \ldots, x_{n}\right] \\
& \leq\left(r_{1} h+\left[x_{2}, \ldots, x_{n}\right]\right)^{*}=\left[r_{1} h, x_{2}, \ldots, x_{n}\right]
\end{aligned}
$$

for $n \geq 1$.

\section{Convergence results}

The key technical condition used to deduce the convergence of continued fractions in $\mathbb{K}$ requires that

$$
\rho_{h}\left(x^{*},(x+t h)^{*}\right) \leq t, \quad t>0,
$$

if $h \leq x$. A weaker variant of this condition is

$$
\rho_{h}\left(x^{*},(x+t h)^{*}\right) \leq C_{R}^{2} t, \quad t>0,
$$

if $h \leq x \leq R h$, where $C_{R}$ is a positive finite function of $R \in[1, \infty]$. Substituting $x=h$ shows that $C_{R} \geq C_{1}=1$. It is immediate that the function $C_{R}$ can be chosen to be non-decreasing and right-continuous. Let $C_{\infty}$ be the constant in the right-hand side of (4) that ensures the inequality for all $x \geq h$. Note that $\rho_{h}\left(x^{*},(x+t h)^{*}\right)=\rho_{h}\left([x],\left[x, t^{-1} h\right]\right)$.

Lemma 3.1. If (4) holds, then

$$
\rho_{h}\left(x^{*}, y^{*}\right) \leq C_{R / r}^{2} r^{-2} \rho_{h}(x, y)
$$

for all $x, y \in \mathbb{K}$ such that $r h \leq x \leq R h$ and $r h \leq y \leq R h$.

Proof. Let $\rho_{h}(x, y) \leq t$. Then $x \leq y+t h$ and $y \leq x+t h$, so that

$$
\rho_{h}\left(x^{*}, y^{*}\right) \leq \max \left(\rho_{h}\left(x^{*},(x+t h)^{*}\right), \rho_{h}\left(y^{*},(y+t h)^{*}\right)\right) .
$$

It suffices to note that (44) and the scaling property of $\rho_{h}$ imply that

$$
\rho_{h}\left(x^{*},(x+t h)^{*}\right) \leq C_{R / r}^{2} r^{-2} t, \quad t>0,
$$

for all $x$ such that $r h \leq x \leq R h$. 
Corollary 3.2. If (4) holds, then the involution operation is $\rho_{h}$-continuous on $\mathbb{K}_{h} \cap \mathbb{K}_{h}^{*}$ and, for $x, y \in \mathbb{K}_{h}$,

$$
\rho_{h}\left(x^{*}, y^{*}\right) \leq C_{\infty}^{2} \max \left(\left\|x^{*}\right\|_{h},\left\|y^{*}\right\|_{h}\right)^{2} \rho_{h}(x, y) .
$$

The following result covers the general case of continued fractions with variable terms.

Theorem 3.3. Assume that $\left(\mathbb{K}_{h}, \rho_{h}\right)$ is complete and condition (4) holds. Assume that there exist $k \geq 1$ and $0<a \leq b \leq \infty$ such that $C_{b / a}<a$ and

$$
\begin{aligned}
x_{n}+\left[x_{n+1}, \ldots, x_{n+2 k}\right] & \geq a h, \\
x_{n}+\left[x_{n+1}, \ldots, x_{n+2 k-1}\right] & \leq b h,
\end{aligned}
$$

for all $n \geq 1$. If, for some $l \geq 0$,

$$
\limsup _{n \rightarrow \infty} \rho_{h}\left(\left[x_{n-2 k-l+1}, \ldots, x_{n}\right],\left[x_{n-2 k-l+1}, \ldots, x_{n+1}\right]\right)<\infty,
$$

then the continued fraction $z_{n}=\left[x_{1}, \ldots, x_{n}\right]$ converges to $z \in \mathbb{K}_{h}$.

Proof. By Lemma 2.4, for all $m \geq 1$ and $n \geq 2 k+m$,

$$
\begin{aligned}
x_{m}+\left[x_{m+1}, \ldots, x_{m+2 k}, x_{m+2 k+1}, \ldots, x_{n}\right] & \geq x_{m}+\left[x_{m+1}, \ldots, x_{m+2 k}, \mathbf{e}^{*}, \ldots, x_{n}\right] \\
& =x_{m}+\left[x_{m+1}, \ldots, x_{m+2 k}\right] \geq a h
\end{aligned}
$$

and

$$
\begin{aligned}
x_{m}+\left[x_{m+1}, \ldots, x_{m+2 k}, x_{m+2 k+1}, \ldots, x_{n}\right] & \leq x_{m}+\left[x_{m+1}, \ldots, x_{m+2 k-1}, \mathbf{e}^{*}, \ldots, x_{n}\right] \\
& =x_{m}+\left[x_{m+1}, \ldots, x_{m+2 k-1}\right] \leq b h .
\end{aligned}
$$

In particular, $z_{n} \leq a^{-1} h$ and so $z_{n} \in \mathbb{K}_{h}$ for sufficiently large $n$. By Lemma 3.1, for $n \geq 2 k+m$,

$$
\begin{aligned}
\rho_{h}\left(\left[x_{m}, \ldots, x_{n}\right],\left[x_{m}, \ldots, x_{n+1}\right]\right) & =\rho_{h}\left(\left(x_{m}+\left[x_{m+1}, \ldots, x_{n}\right]\right)^{*},\left(x_{m}+\left[x_{m+1}, \ldots, x_{n+1}\right]\right)^{*}\right) \\
& \leq C_{b / a}^{2} a^{-2} \rho_{h}\left(\left[x_{m+1}, \ldots, x_{n}\right],\left[x_{m+1}, \ldots, x_{n+1}\right]\right) .
\end{aligned}
$$

By iterating this argument for $m=1, \ldots, n-2 k-l$, we arrive at

$$
\begin{aligned}
\rho_{h}\left(z_{n}, z_{n+1}\right) & \leq\left(a^{-1} C_{b / a}\right)^{2(n-2 k-l)} \rho_{h}\left(\left[x_{n-2 k-l+1}, \ldots, x_{n}\right],\left[x_{n-2 k-l+1}, \ldots, x_{n+1}\right]\right) \\
& \leq\left(a^{-1} C_{b / a}\right)^{2(n-2 k-l)} c
\end{aligned}
$$

for all sufficiently large $n$ and some finite $c$ that dominates the upper limit in (77). Since the series $\sum \rho_{h}\left(z_{n}, z_{n+1}\right)$ converges, the sequence $\left\{z_{n}\right\}$ is fundamental and its convergence follows from the completeness assumption.

Remark 3.4. In Theorem 3.3 it suffices to impose only (5) with $a>C_{\infty}$, which becomes $a>1$ if (3) holds. 
Corollary 3.5. Assume that $\left(\mathbb{K}_{h}, \rho_{h}\right)$ is complete and condition (4) holds. Furthermore, assume that there exist $k \geq 1$ and $0<a \leq b \leq \infty$ such that $q=a^{-1} C_{b / a}<1$, (15) and (6) hold, and there exists $r>0$ such that $x_{n} \geq$ rh for all $n \geq 1$. Then the continued fraction $z_{n}=\left[x_{1}, \ldots, x_{n}\right]$ converges to $z \in \mathbb{K}_{h}$ and

$$
\rho_{h}\left(z_{n}, z\right) \leq \frac{q^{2(n-2 k)}}{1-q^{2}} r^{-1} .
$$

Proof. It suffices to show that the upper limit in (7) for $l=0$ is bounded by $r^{-1}$. Note that $\left[x_{1}, \ldots, x_{n+1}\right]$ lies between $\left[x_{1}, \ldots, x_{n}, \mathbf{e}\right]$ and $\left[x_{1}, \ldots, x_{n}, \mathbf{e}^{*}\right]$, whence

$$
\begin{aligned}
\rho_{h}\left(\left[x_{1}, \ldots, x_{n}\right],\right. & {\left.\left[x_{1}, \ldots, x_{n+1}\right]\right) } \\
& \leq \max \left(\rho_{h}\left(\left[x_{1}, \ldots, x_{n}\right],\left[x_{1}, \ldots, x_{n}, \mathbf{e}\right]\right), \rho_{h}\left(\left[x_{1}, \ldots, x_{n}\right],\left[x_{1}, \ldots, x_{n}, \mathbf{e}^{*}\right]\right)\right. \\
& =\max \left(\rho_{h}\left(\left[x_{1}, \ldots, x_{n}\right],\left[x_{1}, \ldots, x_{n-1}\right]\right), \rho_{h}\left(\left[x_{1}, \ldots, x_{n}\right],\left[x_{1}, \ldots, x_{n}\right]\right)\right. \\
& =\rho_{h}\left(\left[x_{1}, \ldots, x_{n}\right],\left[x_{1}, \ldots, x_{n-1}\right]\right) .
\end{aligned}
$$

By iterating this argument, we obtain

$$
\rho_{h}\left(\left[x_{1}, \ldots, x_{n}\right],\left[x_{1}, \ldots, x_{n+1}\right]\right) \leq \rho_{h}\left(\mathbf{e},\left[x_{1}\right]\right) \leq r^{-1} .
$$

Therefore, $\rho\left(z_{n}, z_{n+1}\right) \leq q^{2(n-2 k)} r^{-1}$, whence

$$
\rho_{h}\left(z_{n}, z_{m}\right) \leq \frac{q^{2(n-2 k)}}{1-q^{2}} r^{-1}
$$

for all $m \geq n$ and it suffices to let $m \rightarrow \infty$.

The subsequent result relies on bounding the terms of the continued fraction by $r h$ from below and $R h$ from above. In the following denote

$$
v(r, R)=[r, R, r, R, \ldots]^{-1}=\frac{1}{2}\left(\sqrt{r^{2}+4 r / R}+r\right), \quad r, R \in(0, \infty],
$$

where the value of the numerical periodic continued fraction is found by solving the recursive equation.

Theorem 3.6. Assume that $\left(\mathbb{K}_{h}, \rho_{h}\right)$ is complete and condition (44) holds. If $r h \leq x_{n} \leq R h$ for all $n \geq 1$ and $0<r \leq R \leq \infty$ such that

$$
\frac{C_{v(R, r) / v(r, R)}}{v(r, R)}<q<1,
$$

then the continued fraction $z_{n}=\left[x_{1}, \ldots, x_{n}\right]$ converges to $z \in \mathbb{K}_{h}$ and (8) holds. 
Proof. By Lemma 2.9,

$$
x_{n}+\left[x_{n+1}, \ldots, x_{n+2 k}\right] \geq(r+[R, r, \ldots, R, r]) h \rightarrow v(r, R) h \quad \text { as } \quad k \rightarrow \infty,
$$

and

$$
x_{n}+\left[x_{n+1}, \ldots, x_{n+2 k-1}\right] \leq(R+[r, R, \ldots, r]) h \rightarrow v(R, r) h \quad \text { as } k \rightarrow \infty .
$$

Fix any $\varepsilon>0$. Then (5) and (6) hold for sufficiently large $k$ with $a=v(r, R)-\varepsilon$ and $b=v(R, r)+\varepsilon$. Finally $C_{b / a}<a$ follows from (10) taking into account that the function $C_{R}$ is right-continuous. The convergence and the bound follow from Corollary 3.5 .

Corollary 3.7. Assume that (3) holds and $\left(\mathbb{K}_{h}, \rho_{h}\right)$ is a complete metric space. Assume that $r h \leq x_{n} \leq R h$ for all $n \geq 1$ with $R \in[r, \infty]$, and either (i) $r>1$ or (ii) $r=1$ and $R<\infty$ or (iii) $r<1$ and $R \leq r /(1-r)$. Then $z_{n}=\left[x_{1}, \ldots, x_{n}\right]$ converges in the metric $\rho_{h}$ to $z \in \mathbb{K}_{h}$ and (8) holds with any $q>v(r, R)$.

Below we present another convergence condition that handles the case when $x_{n} \geq r_{n} h$ with inf $r_{n}=1$.

Theorem 3.8. Assume that (4) holds and $\left(\mathbb{K}_{h}, \rho_{h}\right)$ is complete. Let $\left\{x_{n}, n \geq 1\right\}$ be a sequence of elements from $\mathbb{K}$ such that $r_{n} h \leq x_{n} \leq R_{n} h, n \geq 1$, where $R_{n} \in\left[r_{n}, \infty\right]$. If

$$
\liminf _{n \rightarrow \infty} n \log \left(r_{n} r_{n+1} C_{\left(R_{n}+r_{n+1}^{-1}\right) / r_{n}}^{-2}\right)>1,
$$

then the continued fraction $z_{n}=\left[x_{1}, \ldots, x_{n}\right]$ converges.

Proof. Note that

$$
r_{i} h \leq x_{i}+\left[x_{i+1}, \ldots, x_{n}\right] \leq\left(R_{i}+r_{i+1}^{-1}\right) h
$$

for all $i \leq n-1$. Denote $a_{i}=C_{\left(R_{i}+r_{i+1}^{-1}\right) / r_{i}}, i \geq 1$. By Lemma 3.1 ,

$$
\begin{aligned}
\rho_{h}\left(z_{n}, z_{n+1}\right) & =\rho_{h}\left(\left(x_{1}+\left[x_{2}, \ldots, x_{n}\right]\right)^{*},\left(x_{1}+\left[x_{2}, \ldots, x_{n+1}\right]\right)^{*}\right) \\
& \leq r_{1}^{-2} a_{1}^{2} \rho_{h}\left(\left[x_{2}, \ldots, x_{n}\right],\left[x_{2}, \ldots, x_{n+1}\right]\right) \\
& \leq r_{1}^{-2} r_{2}^{-2} a_{1}^{2} a_{2}^{2} \rho_{h}\left(\left[x_{3}, \ldots, x_{n}\right],\left[x_{3}, \ldots, x_{n+1}\right]\right) \\
& \leq \rho_{h}\left(x_{n}^{*},\left(x_{n}+x_{n+1}^{*}\right)^{*}\right) \prod_{i=1}^{n-1}\left(r_{i}^{-2} a_{i}^{2}\right) \leq r_{n+1}^{-1} \prod_{i=1}^{n}\left(r_{i}^{-2} a_{i}^{2}\right) .
\end{aligned}
$$

In view of (11), the logarithmic convergence criterion yields that the series $\sum \rho_{h}\left(z_{n}, z_{n+1}\right)$ converges, so that the sequence $\left\{z_{n}\right\}$ is fundamental.

Remark 3.9. If (3) holds, then (11) becomes

$$
\liminf _{n \rightarrow \infty} n \log \left(r_{n} r_{n+1}\right)>1
$$


Theorem 3.6 and Corollary 3.7 cover the important case of continued fractions

$$
z_{n}=[\underbrace{x, \ldots, x}_{n}]
$$

with constant terms. An alternative proof of the convergence of continued fractions with constant terms under the same conditions can be carried over using the contraction mapping theorem.

For continued fractions with constant terms, it may be of advantage to check directly the conditions of Theorem 3.3 instead of bounding the term from below and from above.

Corollary 3.10. Assume that $\left(\mathbb{K}_{h}, \rho_{h}\right)$ is complete and condition (4) holds. If $x \in \mathbb{K}_{h}^{*}$ is such that, for some $k \geq 1$,

$$
x+[\underbrace{x, \ldots, x}_{2 k}] \geq a h
$$

and

$$
x+[\underbrace{x, \ldots, x}_{2 k-1}] \leq b h
$$

with $a>C_{b / a}$, then the continued fraction (12) converges.

Corollary 3.11. Assume that $\left(\mathbb{K}_{h}, \rho_{h}\right)$ is complete and condition (3) holds. Let $z_{n}$ be the nth approximant of the continued fraction with constant term $x \in \mathbb{K}_{h}^{*}$. If $z_{2 k-1} \leq$ rh for $r<1$ and some $k \geq 1$, then $z_{n}$ converges to $z \in \mathbb{K}_{h}$.

Remark 3.12. The limit $z$ of the continued fraction with constant term $x$ satisfies the equation

$$
z^{*}=z+x,
$$

Consider now the changes that happen to the basic equation (13) if either $z$ or $x$ are scaled. For each $t \geq 1, t^{-1} z$ satisfies

$$
\left(t^{-1} z\right)^{*}=t^{-1} z+x_{t}
$$

for $x_{t}=\left(t-t^{-1}\right) z^{*}+t^{-1} x$. Indeed,

$$
t^{-1} z+x_{t}=t^{-1}(z+x)+\left(t-t^{-1}\right) z^{*}=t z^{*}=\left(t^{-1} z\right)^{*} .
$$

Assume that (3) holds and $r h \leq x \leq R h$ so that one of the conditions of Corollary 3.7 holds. Then the continued fraction with the constant term $t x$ converges for all $t \geq 1$, so that there exists unique $z_{t} \in \mathbb{K}$ that satisfies

$$
z_{t}^{*}=z_{t}+t x, \quad t \geq 1 .
$$

Fix $\beta \in[0,1]$ and note that $y_{t}=t^{\beta} z_{t}$ satisfies the equation

$$
y_{t}^{*}=t^{-2 \beta} y_{t}+t^{1-\beta} x .
$$


Since $x \geq r h$, we have $y_{t} \leq t^{\beta-1} r h$. If $\beta<1$, then $y_{t}=t^{\beta} z_{t}$ converges to e as $t \rightarrow \infty$ by Lemma 2.5. If $\beta=1$, then the equation becomes $y_{t}^{*}=t^{-2} y_{t}+x$. Since

$$
t^{-2} y_{t}=t^{-1} z_{t}=t^{-1}\left(z_{t}+t x\right)^{*} \leq t^{-2} x^{*} \leq t^{-2} r^{-1} h
$$

we have $\left(x+t^{-2} r^{-1} h\right)^{*} \leq t z_{t} \leq x^{*}$. Therefore, $t z_{t}$ converges to $x$ as $t \rightarrow \infty$.

While the following result can be proved under condition (44), we formulate its simpler version.

Theorem 3.13. Assume that (3) holds. If the continued fractions with constant terms $x^{\prime}$ and $x^{\prime \prime}$, such that $x^{\prime} \geq r h$ and $x^{\prime \prime} \geq r h$ with $r>1$, converge respectively to $z^{\prime}$ and $z^{\prime \prime}$, then

$$
\rho_{h}\left(z^{\prime}, z^{\prime \prime}\right) \leq \rho_{h}\left(x^{\prime}, x^{\prime \prime}\right) \frac{1}{r^{2}-1} .
$$

Proof. It follows from (13), the triangle inequality, the translation invariance of the metric $\rho_{h}$, and Lemma 3.1 that

$$
\begin{aligned}
\rho_{h}\left(z^{\prime}, z^{\prime \prime}\right) & =\rho_{h}\left(\left(z^{\prime}+x^{\prime}\right)^{*},\left(z^{\prime \prime}+x^{\prime \prime}\right)^{*}\right) \\
& \leq r^{-2} \rho_{h}\left(z^{\prime}+x^{\prime}, z^{\prime \prime}+x^{\prime \prime}\right) \\
& \leq r^{-2}\left(\rho_{h}\left(z^{\prime}, z^{\prime \prime}\right)+\rho_{h}\left(x^{\prime}, x^{\prime \prime}\right)\right)
\end{aligned}
$$

The statement is obtained after rearranging the terms taking into account that $r>1$.

Example 3.14. The setting of Theorem 3.3 is well adjusted to confirm the convergence of periodic continued fractions. For instance, consider the continued fraction with alternating elements $x, y \in \mathbb{K}$ assuming that (3) holds. If $x \geq \varepsilon h, y \geq \varepsilon h$ for some $\varepsilon>0$, and

$$
x+\left(y+x^{*}\right)^{*} \geq r h, \quad y+\left(x+y^{*}\right)^{*} \geq r h
$$

for some $r>1$, then (5) holds with $k=1$ and the continued fraction $[x, y, x, y, \ldots]$ converges. If $y=x^{*}$, and (3) holds, then condition (15) for sufficiently large $k$ amounts to $v(1,1) x \geq r h$ and $v(1,1) x^{*} \geq r h$ for some $r>1$, where $v(1,1)=\frac{1}{2}(1+\sqrt{5})$. In this case, the continued fraction $\left[x, x^{*}, x, x^{*}, \ldots\right]$ converges and the polar $y=z^{*}$ to its limit $z$ satisfies the equation

$$
x+(x \oplus y)=y .
$$

\section{Continued fractions of convex sets}

Let $\mathbb{K}=\mathcal{C}_{0}$ be the family of all convex closed sets in $\mathbb{R}^{d}$ containing the origin. We refer to [11] for a wealth of information about convex sets. The closed Minkowski sum $K+L$ of two sets $K, L \in \mathcal{C}_{0}$ is defined as the closure of the set $\{x+y: x \in K, y \in L\}$ of pairwise sums of points from $K$ and $L$. If at least one summand is compact, then the set of pairwise sums is closed and no additional closure is required. 
The family $\mathcal{C}_{0}$ with the closed Minkowski addition is a semigroup with the neutral element $\mathbf{e}=\{0\}$ being the origin, partially ordered by inclusion. Note that all inclusions for sets are understood in the non-strict sense. Since the convex sets from $\mathcal{C}_{0}$ contain the origin, $K \subset L$ implies that $K+M \subset L+M$ for $K, L, M \in \mathcal{C}_{0}$, so that the order is compatible with the addition. The scaling by positive reals is defined conventionally as $a K=\{a x: x \in K\}$.

It is known that the only order reversing involution on $\mathcal{C}_{0}$ (up to a rigid motion) is the polar transform, see [1, 5]. The polar to $K \in \mathcal{C}_{0}$ is defined as

$$
K^{*}=\left\{u \in \mathbb{R}^{d}: s_{K}(u) \leq 1\right\},
$$

where

$$
s_{K}(u)=\sup \{\langle u, x\rangle: x \in K\}
$$

is the support function of $K$ and $\langle u, x\rangle$ denotes the scalar product. Note that the support function may take infinite values if $K$ is not bounded. Since the support function is homogeneous of order 1, it suffices to consider its values for $u$ with the Euclidean norm $\|u\|=1$, i.e. for all $u$ from the unit Euclidean sphere $\mathbb{S}^{d-1}$ in $\mathbb{R}^{d}$. The inclusion of convex sets turns into the pointwise domination of their support functions. Let

$$
r_{K}(u)=\sup \{t: t u \in K\}
$$

be the radial function of $K \in \mathcal{C}_{0}$. Then $r_{K^{*}}(u)=1 / s_{K}(u)$ for all unit vectors $u$, see [11, Sec. 1.6].

The only convex set invariant for the polar transform is the unit Euclidean ball $B$. If $K=r B$ is the ball of radius $r$ centred at the origin, then $K^{*}=r^{-1} B$ is the ball of radius $r^{-1}$. Further examples can be found in [11, Sec. 1.6]. The polar to the neutral element $\{0\}$ is the whole space, i.e. $\mathbf{e}^{*}=\mathbb{R}^{d}$.

The family $\mathbb{K}_{h}=\mathcal{K}_{0}$ consists of convex bodies (i.e. convex compact sets) containing the origin and the metric $\rho_{h}$ from (1) is the Hausdorff distance

$$
\rho_{H}(K, L)=\inf \{\varepsilon>0: K \subset L+\varepsilon B, L \subset K+\varepsilon B\}
$$

between $K$ and $L$ from $\mathcal{K}_{0}$. Note that $K+\varepsilon B$ is called the $\varepsilon$-envelope of $K$, which is alternatively defined as the set of points within distance at most $\varepsilon$ to $K$. The norm of a set defined as

$$
\|K\|=\sup \{\|x\|: x \in K\}=\rho_{H}(K,\{0\})
$$

is the radius of the smallest centred ball that contains $K$. The family $\mathbb{K}_{h}^{*}$ consists of all convex closed sets that contain a neighbourhood of the origin and $\mathbb{K}_{h} \cap \mathbb{K}_{h}^{*}=\mathcal{K}_{00}$ is the family of convex bodies containing a neighbourhood of the origin.

It is known that $\mathcal{K}_{0}$ with the Hausdorff metric is a complete separable metric space, see [11, Th. 1.8.3]. We apply the same definition of the Hausdorff metric also for closed (possibly non-compact) sets, noticing that $\rho_{H}$ may take infinite values.

Consider the set-valued continued fraction given by

$$
F_{n}=\left[K_{1}, \ldots, K_{n}\right], \quad n \geq 1,
$$

for a sequence $K_{n} \in \mathcal{C}_{0}, n \geq 1$. 
Theorem 4.1. Let $K_{n}=K$ for all $n \geq 1$ and $K \in \mathcal{C}_{0}$. Assume that $K \supset r B$, where either (i) $r>1$ or (ii) $r=1$ and $K$ is compact or (iii) $0<r<1$ and $\|K\|<r /(1-r)$. Then

$$
F_{n}=[\underbrace{K, \ldots, K}_{n}]
$$

converges in the Hausdorff metric to a convex body $F \in \mathcal{K}_{0}$ that satisfies the equation

$$
F^{*}=F+K
$$

Proof. We confirm the validity of (3) that becomes

$$
\rho_{H}\left(K^{*},(K+t B)^{*}\right) \leq t
$$

for all $K \in \mathcal{C}_{0}$ such that $B \subset K$. Then $K^{*} \subset B$ and

$$
\begin{aligned}
K^{*} & =\left\{u \in B: s_{K}(u) \leq 1\right\} \\
& =\left\{u \in B: s_{K}(u)+s_{t B}(u) \leq 1+t\|u\|\right\} \\
& \subset\left\{u \in B: s_{K}(u)+s_{t B}(u) \leq 1+t\right\} \\
& =(1+t)\left\{u \in(1+t)^{-1} B: s_{K}(u)+s_{t B}(u) \leq 1\right\} \\
& \subset(1+t)\left\{u \in B: s_{K+t B}(u) \leq 1\right\} \\
& =(1+t)(K+t B)^{*} .
\end{aligned}
$$

For any convex body $A$ and any $\varepsilon>0$,

$$
(1+\varepsilon) A \subset A+\varepsilon\|A\| B .
$$

Indeed, taking the support functions of the both sides, it is immediately seen that

$$
(1+\varepsilon) s_{A}(u) \leq s_{A}(u)+\varepsilon\|A\|, \quad u \in \mathbb{S}^{d-1} .
$$

In view of this and the fact that $(K+t B)^{*} \subset B$,

$$
K^{*} \subset(1+t)(K+t B)^{*} \subset(K+t B)^{*}+t B,
$$

so that (16) holds, and Corollary 3.7 yields the result.

In view of Corollary [3.2, condition (3) verified in the proof of Theorem 4.1 yields the following result that is of independent interest.

Theorem 4.2. For any two convex compact sets $K, L$ containing the origin,

$$
\rho_{H}\left(K^{*}, L^{*}\right) \leq \max \left(\left\|K^{*}\right\|,\left\|L^{*}\right\|\right)^{2} \rho_{H}(K, L) .
$$

Theorem 4.3. Let $K$ be a convex set containing a neighbourhood of the origin. The continued fraction (14) with constant term $K$ converges in the Hausdorff metric if and only if $F_{2 k-1} \subset a B$ for $a<1$ and at least one $k \geq 1$. 
Proof. The sufficiency follows from Corollary 3.11. Assume that $F_{n}$ converges to $F$ that necessarily satisfies (15). Let $K \supset \varepsilon B$ for $\varepsilon>0$. Then $F \subset \varepsilon^{-1} B$, whence $F$ is also compact and $\|F\|=R$ is finite. Then there exists $u$ with $\|u\|=1$ such that $s_{F}(u)=r_{F}(u)=R$. It follows from (15) that

$$
s_{F^{*}}(u) \geq s_{F}(u)+\varepsilon .
$$

Since $s_{F^{*}}(u)=1 / r_{F}(u)=1 / s_{F}(u)$, we have

$$
\frac{1}{s_{F}(u)} \geq s_{F}(u)+\varepsilon,
$$

whence

$$
R=s_{F}(u)=\frac{1}{2}\left(\sqrt{\varepsilon^{2}+4}-\varepsilon\right)<1 .
$$

Thus, $F_{n} \subset a B$ for $a \in(R, 1)$ and all sufficiently large $n$, in particularly, for the oddnumbered terms.

Remark 4.4. Since the sequences $F_{2 n}$ and $F_{2 n-1}$ for the set-valued continued fraction with the constant term $K$ are monotone and bounded, they converge without any extra condition on the set $K$. But their limits may be different.

Example 4.5. a) It is easy to see that $K=r B$ satisfies the condition of Theorem 4.1 for each $r>0$.

b) A segment $K=[0, u]$ for a given $u \in \mathbb{R}^{d}$ does not satisfy the condition and the corresponding continued fraction diverges. Indeed, $F_{n}$ is a segment for even $n$ and a halfspace for odd $n$.

c) Assume that $K$ is a strip $\left\{\left(x_{1}, x_{2}\right):-a \leq x_{1} \leq a\right\} \subset \mathbb{R}^{2}$. Then $K^{*}$ is the segment with end-points at $\left( \pm a^{-1}, 0\right)$. Thus, $F_{n}$ is the segment with end-points at $\left( \pm a_{n}, 0\right)$ where $a_{n}=[a, \ldots, a]$. Thus, $F_{n}$ converges, whereas $K$ does not satisfy the condition of Theorem 4.1 if $a \leq 1$. However, $F_{2 k+1} \subset r B$ with $r<1$ for sufficiently large $k$, and so Theorem 4.3 confirms the convergence of the continued fraction.

In relation to continued fractions generated by non-constant sequences, Theorem 3.3 taking into account Remark 3.4 applies. Furthermore, the continued fraction converges if $r B \subset K_{n} \subset R B$ for all $n \geq 1$ and for $r$ and $R$ satisfying the conditions of Corollary [3.7. By Theorem 3.8, the continued fraction converges if $r_{n} B \subset K_{n}$ and $\lim \inf _{n \rightarrow \infty} n \log \left(r_{n} r_{n+1}\right)>1$.

Example 4.6. Let $K$ and $L$ be two different centred segments in the plane. Then the infinite sum $K+L+K+L+\cdots$ is the whole plane. However, the continued fraction $[K, L, K, L, \ldots]$ diverges. Indeed, $K^{*}$ is a strip, so that $L+K^{*}$ is the same strip of a different width, so that $\left(L+K^{*}\right)^{*}$ if a scaled variant of $K$, and the successive iterations result in a sequence that alternates between a scale of $K$ and a polar to it. This example shows that a direct generalisation of the Seidel-Stern theorem on continued fractions with positive terms [ 6 , Th. 4.28] fails in the set-valued case.

Example 4.7. Convex sets that appear as limits of periodic continued fractions might be regarded as a generalisation of quadratic irrational numbers. Consider the continued fraction 
$[K, L, K, L, \ldots]$ with two alternating terms $K, L \in \mathcal{C}_{0}$. By Corollary 3.5 together with Remark 3.4, this continued fraction converges if $K+\left(L+K^{*}\right)^{*} \supset a B$ and $L+\left(K+L^{*}\right)^{*} \supset a B$ for $a>1$, and both $K$ and $L$ contain a neighbourhood of the origin.

Example 4.8. In the setting of Example 4.7, the continued fraction diverges if $K$ and $L$ are two segments, see also Example 4.6. In order to obtain a converging continued fraction built of segments, consider three centred non-collinear segments $L_{i}=\left[-u_{i}, u_{i}\right], i=1,2,3$, in the plane and the corresponding continued fraction $\left[K_{1}, K_{2}, \ldots\right]$, where $K_{3 n+i}=\left[-v_{3 n+i}, v_{3 n+i}\right]=$ $L_{i}$ for $n \geq 0$ and $i=1,2,3$. Condition (5) for $k=1$ amounts to

$$
K_{n}+\left(K_{n+1}+K_{n+2}^{*}\right)^{*} \supset a B
$$

with $a>1$. A direct geometric calculation shows that

$$
\left(K_{n+1}+K_{n+2}^{*}\right)^{*}=\frac{1}{1+\left|\left\langle v_{n+1}, v_{n+2}\right\rangle\right|}\left[-v_{n+2}, v_{n+2}\right] .
$$

Thus, (5) holds if

$$
\left[-u_{i_{1}}, u_{i_{1}}\right]+\frac{1}{1+\left|\left\langle u_{i_{2}}, u_{i_{3}}\right\rangle\right|}\left[-u_{i_{3}}, u_{i_{3}}\right] \supset a B
$$

for some $a>1$ and all permutations $\left(i_{1}, i_{2}, i_{3}\right)$ of $(1,2,3)$. This is always possible to achieve by increasing the lengths of the segments. Furthermore, (7) holds with $l=1$ if

$$
\left[K_{n-2 k}, \ldots, K_{n}\right] \subset R B, \quad\left[K_{n-2 k}, \ldots, K_{n+1}\right] \subset R B,
$$

for a finite fixed $R$ and all sufficiently large $n$. In case of segments, this condition holds, since each of the approximants in (18) take only three possible values and all they are compact, since

$$
\left[K_{n}, K_{n+1}, K_{n+2}\right]^{*}=K_{n}+\left(K_{n+1}+K_{n+2}^{*}\right)^{*}
$$

contains a neighbourhood of the origin.

Remark 4.9. The Minkowski sum in the definition of set-valued continued fractions can be replaced by other operations with sets, e.g. the convex hull of the union, the $L_{p^{-}}$sum or the radial sum, see [11. For instance, if the convex hull of the union $K \vee M=\operatorname{conv}(K \cup M)$ is chosen as the semigroup operation, then $K \vee t B \subset K+t B$, so that (3) holds in this case and the convergence results from Section 2 apply. Furthermore, it is possible to consider a sequence of alternating operations, e.g. the Minkowski sum and the radial sum. The latter case is particularly easy, since its reduces to the numerical continued fraction built of the values of the support function of the terms, so that the classical convergence criteria apply.

\section{Space of non-negative convex functions}

Let $\mathbb{K}=\mathrm{Cvx}_{0}\left(\mathbb{R}^{d}\right)$ be the space of convex functions $f: \mathbb{R}^{d} \mapsto[0, \infty]$ such that $f(0)=0$ with the arithmetic addition as the semigroup operation and the pointwise partial order. The Legendre-Fenchel transform of a function $f$ is defined as

$$
f^{*}(x)=\sup _{y}(\langle x, y\rangle-f(y)), \quad x \in \mathbb{R}^{d} .
$$


It is well known that the Legendre-Fenchel transform is an order reversing involution on $\operatorname{Cvx}_{0}\left(\mathbb{R}^{d}\right)$, see [9]. The neutral element is the function identically equal to zero, and its dual is the convex function identically equal to infinity outside the origin. The only self-polar function is $h(x)=\frac{1}{2}\|x\|^{2}$. The family $\mathbb{K}_{h}$ is the family of convex functions that admit a quadratic majorant, and the metric $\rho_{h}$ is given by

$$
\rho_{h}(f, g)=\inf \left\{\varepsilon>0: f(x) \leq g(x)+\frac{\varepsilon}{2}\|x\|^{2}, g(x) \leq f(x)+\frac{\varepsilon}{2}\|x\|^{2}, x \in \mathbb{R}^{d}\right\} .
$$

It is easy to see that (2) holds and so $\rho_{h}$ is indeed a metric.

The dual operation (see Remark 2.1) to the arithmetic addition is the inf-convolution

$$
(f \oplus g)(x)=\inf _{x_{1}+x_{2}=x}\left(f\left(x_{1}\right)+g\left(x_{2}\right)\right) .
$$

Consider the continued fraction

$$
z_{n}=\left[f_{1}, \ldots, f_{n}\right] \in \operatorname{Cvx}_{0}\left(\mathbb{R}^{d}\right), \quad n \geq 1,
$$

generated by a sequence $\left\{f_{n}, n \geq 1\right\}$ from $\mathrm{Cvx}_{0}\left(\mathbb{R}^{d}\right)$.

Theorem 5.1. Assume that a function $f \in \mathrm{Cvx}_{0}\left(\mathbb{R}^{d}\right)$ satisfies $\frac{r}{2}\|x\|^{2} \leq f(x) \leq \frac{R}{2}\|x\|^{2}$ for all $x \in \mathbb{R}^{d}$, where $R \in[r, \infty]$, and

$$
r^{2}+\frac{4 r}{R}>4
$$

Then the continued fraction $z_{n}=[f, \ldots, f]$ with the constant term $f$ converges in the metric $\rho_{h}$ to $z \in \mathbb{K}_{h}$ satisfying $z^{*}=z+f$.

Proof. It is easy to see that (4) holds with $C_{R} \leq C_{\infty}=2$. Indeed, if $f \geq h$, then

$$
\langle x, x+v\rangle-f(x+v) \leq\langle x, x+v\rangle-\frac{1}{2}\|x+v\|^{2}=\frac{1}{2}\|x\|^{2}-\frac{1}{2}\|v\|^{2},
$$

whence

$$
\begin{aligned}
f^{*}(x) & =\sup _{v \in \mathbb{R}^{d}}[\langle x, x+v\rangle-f(x+v)] \\
& =\sup _{\|v\| \leq\|x\|}[\langle x, x+v\rangle-f(x+v)] \\
& =\sup _{\|v\| \leq\|x\|}\left[\langle x, x+v\rangle-f(x+v)-\frac{1}{2} t\|x+v\|^{2}+\frac{1}{2} t\|x+v\|^{2}\right] \\
& \leq \sup _{v \in \mathbb{R}^{d}}\left[\langle x, x+v\rangle-f(x+v)-\frac{1}{2} t\|x+v\|^{2}+2 t\|x\|^{2}\right] \\
& =(f+t h)^{*}(x)+4 t h .
\end{aligned}
$$

Thus, $\rho_{h}\left(f^{*},(f+t h)^{*}\right) \leq 4 t$. In order to improve the inequality, start by observing that

$$
\begin{aligned}
\rho_{h}\left(f^{*},(f+t h)^{*}\right) & =\inf \left\{\varepsilon>0: f^{*} \leq(f+t h)^{*}+\varepsilon h\right\} \\
& =\inf \left\{\varepsilon>0: f^{*} \leq\left(f^{*} \oplus t^{-1} h\right)+\varepsilon h\right\} .
\end{aligned}
$$


If $h \leq f \leq R h$, then $R^{-1} h \leq f^{*} \leq h$. Therefore,

$$
f^{*}(x-y) \geq f^{*}(x)-k_{x}\|y\|
$$

where $k_{x}=\|x\|\left(1+\sqrt{1-R^{-1}}\right)$. Indeed, $k_{x}$ is the steepest slope of the tangent line to the graph of $\frac{1}{2}\|u\|^{2}, u \in \mathbb{R}^{d}$, that passes through the point $\left(x, \frac{1}{2} R^{-1}\|x\|^{2}\right) \in \mathbb{R}^{d+1}$. Thus,

$$
\begin{aligned}
\left(f^{*} \oplus t^{-1} h\right)(x) & =\inf \left(f^{*}(x-y)+\frac{1}{2} t^{-1}\|y\|^{2}\right) \\
& \geq f^{*}(x)+\inf \left(-k_{x}\|y\|+\frac{1}{2} t^{-1}\|y\|^{2}\right) \\
& \geq f^{*}(x)-\frac{1}{2} t\|x\|^{2}\left(1+\sqrt{1-R^{-1}}\right)^{2}
\end{aligned}
$$

Thus, (4) holds with

$$
C_{R}=1+\sqrt{1-R^{-1}}
$$

Finally, (10) holds if

$$
1+\sqrt{1-\frac{v(r, R)}{v(R, r)}}<v(r, R)
$$

which is equivalent to the imposed condition (19).

It remains to show that $\mathbb{K}_{h}$ is complete in the metric $\rho_{h}$. If $\left\{f_{n}\right\}$ is a fundamental sequence in $\rho_{h}$, then $f_{n}(x)$ is a fundamental sequence for each $x \in \mathbb{R}^{d}$, so that $f_{n}(x) \rightarrow f(x)$ for all $x$ and $f \in \mathbb{K}_{h}$. Finally, $f_{n} \leq f_{m}+\varepsilon h$ for any $\varepsilon>0$ and all sufficiently large $n$ and $m$ implies that $f \leq f_{m}+\varepsilon h$ and $f_{n} \leq f+\varepsilon h$ by letting $n \rightarrow \infty$ and $m \rightarrow \infty$.

Given that (4) holds with $C_{R}$ given by (20), all results from Section 3 can be used to obtain further sufficient conditions for the convergence of continued fractions in $\operatorname{Cvx}_{0}\left(\mathbb{R}^{d}\right)$. For instance, Corollary 3.10 implies that the continued fraction with constant term $f$ converges if its approximants satisfy $z_{2 k+1} \leq a^{-1} h$ and $z_{2 k} \geq b^{-1} h$ for some $k \geq 1$ with $a>C_{b / a}$. The latter condition amounts to $a>2-b^{-1}$.

The other polarity transform (A-transform) on $\operatorname{Cvx}_{0}\left(\mathbb{R}^{d}\right)$ thoroughly analysed in [3] is given by

$$
f^{o}(x)= \begin{cases}\sup \left\{\frac{\langle x, y\rangle-1}{f(y)}: y \in \mathbb{R}^{d}, f(y)>0\right\}, & x \in\left\{f^{-1}(0)\right\}^{*} \\ \infty, & \text { otherwise }\end{cases}
$$

For simplicity, assume that $d=1$. In this case the family of self-polar functions includes

$$
h_{p}(x)=\left(\frac{(p-1)^{p-1}}{p^{p}}\right)^{\frac{1}{2}}\|x\|^{p}
$$

for any $p \in[1, \infty]$, see [10]. 
For any finite positive outside the origin self-polar function $h$, condition (3) holds. Indeed, if $f \geq h$, then

$$
\begin{aligned}
f^{o}(x) & =\sup _{y \neq 0}\left[\frac{\langle x, y\rangle-1}{f(y)}-\frac{\langle x, y\rangle-1}{f(y)+t h(y)}+\frac{\langle x, y\rangle-1}{f(y)+t h(y)}\right] \\
& \leq \sup _{y \neq 0} \frac{t h(y)(\langle x, y\rangle-1)}{f(y)(f(y)+t h(y))}+(f+t h)^{*}(x) \\
& \leq t \sup _{y \neq 0} \frac{\langle x, y\rangle-1}{h(y)}+(f+t h)^{*}(x) \\
& =\operatorname{th}(x)+(f+t h)^{*}(x) .
\end{aligned}
$$

Since $\mathbb{K}_{h}$ is complete with the $\rho_{h}$ metric, Corollary 3.7 yields the convergence of continued fractions with constant terms and further results from Section 3 apply in this case.

\section{Acknowledgements}

The author is grateful to the Department of Statistics of the University Carlos III of Madrid for hospitality in 2012 when this work commenced and to Bernardo d'Auria for numerous discussions at early stages. The author has also benefited from discussions with Sergei Foss, Daniel Hug, Takis Konstantopoulos and Matthias Reitzner.

\section{References}

[1] S. Artstein-Avidan and V. Milman. The concept of duality for measure projections of convex bodies. J. Funct. Anal., 254:2648-2666, 2008.

[2] S. Artstein-Avidan and V. Milman. The concept of duality in convex analysis, and the characterization of the Legendre transform. Ann. of Math. (2), 169(2):661-674, 2009.

[3] S. Artstein-Avidan and V. Milman. Hidden structures in the class of convex functions and a new duality transform. J. Eur. Math. Soc. (JEMS), 13(4):975-1004, 2011.

[4] E. Bernadac. Random continued fractions and inverse Gaussian distribution on a symmetric cone. J. Theoret. Probab., 8:221-259, 1995.

[5] K. J. Böröczky and R. Schneider. A characterization of the duality mapping for convex bodies. Geom. Funct. Anal., 18(3):657-667, 2008.

[6] W. B. Jones and W. J. Thron. Continued Fractions, volume 11 of Encyclopedia of Mathematics and its Applications. Addison-Wesley Publishing Co., Reading, Mass., 1980. Analytic theory and applications, With a foreword by Felix E. Browder, With an introduction by Peter Henrici. 
[7] O. Karpenkov. Geometry of Continued Fractions, volume 26 of Algorithms and Computation in Mathematics. Springer, Heidelberg, 2013.

[8] M. L. Kontsevich and Y. M. Suhov. Statistics of Klein polyhedra and multidimensional continued fractions. In Pseudoperiodic topology, volume 197 of Amer. Math. Soc. Transl. Ser. 2, pages 9-27. Amer. Math. Soc., Providence, RI, 1999.

[9] R. T. Rockafellar. Convex Analysis. Princeton University Press, Princeton, NJ, 1970.

[10] L. Rotem. Characterization of self-polar convex functions. Bull. Sci. Math., 136(7):831$838,2012$.

[11] R. Schneider. Convex Bodies. The Brunn-Minkowski Theory. Cambridge University Press, Cambridge, 2 edition, 2014. 